\title{
Functional polyaniline nanofibre mats for human adipose-derived stem cell proliferation and adhesion
}

\begin{abstract}
Conductive polymer poly(aniline-co-m-aminobenzoic acid) (P(ANI-co-m-ABA)) and polyaniline (PANI) were blended with a biodegradable, biocompatible polymer, poly(l-lactic acid) and were electrospun into nanofibres to investigate their potential application as a scaffold for human adipose-derived stem cells (hASCs). These polymers, in both conductive and non-conductive form, were electrospun with average fibre diameters of less than $400 \mathrm{~nm}$. Novel nanoindentation results obtained on the individual nanofibres revealed that the elastic moduli of the nanofibres are much higher at the surface (4-10 GPa, hmax $<75 \mathrm{~nm}$ ) than in the inner fibre core $(2-4 \mathrm{GPa}$, hmax $>75 \mathrm{~nm})$. The composite nanofibres showed great promise as a scaffold for hASCs as they supported the cell adhesion and proliferation. After 1 week of cell culture hASCs were well spread on the substrates with abundant focal adhesions. The electrospun mats provide the cells with comparably stiff, sub-micron sized fibres as anchoring points on a substrate of high porosity. The conductive nature of these composite nanofibres offers exciting opportunities for electrical stimulation of the cells.
\end{abstract}

Keyword: Biomaterials; Electronic materials; Nanostructures; Polymers. 